\title{
EL APRENDIZAJE-SERVICIO COMO ESTRATEGIA DIDÁCTICA PARA LA PROFESIONALIZACIÓN, LA SOSTENIBILIDAD Y LA TRANSFORMACIÓN SOCIAL. UNA EXPERIENCIA EN EL GRADO DE EDUCACIÓN SOCIAL
}

\author{
SERVICE-LEARNING AS A DIDACTIC STRATEGY FOR \\ PROFESSIONALIZATION, SUSTAINABILITY AND SOCIAL \\ TRANSFORMATION. AN EXPERIENCE IN THE DEGREE OF SOCIAL \\ EDUCATION
}

\author{
Ma Gloria Solís Galán, Concepción López Andrada
}

Universidad de Extremadura

\author{
Correspondencia: $M^{\mathrm{a}}$ Gloria Solís Galán \\ Correo: glsolisg@unex.es \\ Recibido: 01/03/20; Aceptado: 09/06/2020 \\ DOI: 10.17398/0213-9529.40.1.73
}

\begin{abstract}
RESUMEN
La Educación Superior tiene como tarea formar a los y las profesionales que la sociedad necesita en cada momento, especialización y sector; por otra parte, favorecer su inserción laboral y empleabilidad. Profesionales y ciudadanos/as comprometidos que intervienen y actúan en la realidad como agentes de transformación y cambio social hacia la sostenibilidad. El propósito de este trabajo es contextualizar y describir un proyecto de Aprendizaje-Servicio en el grado de Educación Social (Universidad de Extremadura) orientado a la adquisición de competencias profesionalizantes mediante la participación activa del alumnado en una experiencia real asociada al servicio comunitario. A través de este aprendizaje experiencial, el estudiante explora potencialidades de mejora de las entidades del Tercer Sector no lucrativo de bienestar social, las cuales trabajan en su entorno próximo, a la vez que desarrollan competencias genéricas y específicas necesarias para el desempeño laboral, y para el ejercicio de una ciudadanía participativa y solidaria.
\end{abstract}

Palabras clave: aprendizaje-servicio; educación superior; tercer sector; justicia social

\begin{abstract}
The role of Higher Education is to train the professionals that society needs at each moment, specialization and sector; on the other hand, to favor their labor insertion and/or employability. Professionals and committed citizens who intervene and act in reality as agents of transformation and social change towards sustainability. The purpose of this paper is to contextualize and describe a Service-Learning project in the Social Education degree (University of Extremadura) aimed at the acquisition of professional skills through the active participation of students in a real experience associated with community service. Through this experiential learning the student explores the potential for improvement of the non-profit welfare entities of the Third Sector, which work in their close environment, while developing generic and specific skills necessary for job performance, and for the exercise of a participative and supportive citizenship.
\end{abstract}

Keyword: service-learning; higher education; third sector; social justice. 


\section{INTRODUCCIÓN}

\section{Resignificando roles, espacios y equilibrios para el análisis y la transformación social}

En las sociedades contemporáneas el sujeto sufre un proceso de "individualización" descrito como la desvinculación de este con las formas tradicionalmente comunitarias (Beck, 1998). En cambio, habitamos una época cambiante que se singulariza por esa "modernidad líquida" (Bauman, 2007) que impregna nuestras relaciones con los otros; por la denominada "sociedad del riesgo" y por las lógicas mercantiles. Relaciones de desigualdad que se establecen en el tardocapitalismo, intensificadas en los periodos de crisis, producidas en el interior de su estructura, "un orden al que no sólo se fuerza a la gente, a los cuerpos y a las cosas, sino en el que, además, estos juegan simultáneamente un papel activo" (Lorey 2008, p. 63-64); en consecuencia, el estudiante -el futuro trabajador- debe ajustarse a un mundo competitivo mediado por el capital en el que "las ideas de autonomía y libertad están constitutivamente conectadas con los modos hegemónicos de subjetivación en las sociedades capitalistas occidentales" (Lorey 2008, p. 58). Cobrará, entonces, mayor relevancia la práctica educativa desde el ejercicio de la crítica y de la transformación política- y la resignificación de la figura y funciones del Educador Social - como agente de cambio activo y autoconsciente-.

Desde diversas latitudes (Gaete, 2015; Gallardo, 2017; López-Rodríguez, 2017) se está impulsando el denominado "emprendimiento social" como dispositivo transformador y de contrapoder ante los desafíos que emergen en el campo político-social y que serán necesarios incorporar a los espacios educativos y a las reflexiones sobre la construcción de una ciudadanía crítica. Por lo tanto, la comunidad educativa entendida desde lo integral y lo global amplias vías de transmisión y de estructuración de las bases del emprendimiento social (López-Rodríguez, 2017).

El emprendimiento social proporciona valor social sostenible (Moreira y Urriolagoitia, 2011; citado en García-Hernández et al, 2017), es decir, en palabras de Marchante y Sánchez (2015):

La generación de valor social por parte de los emprendimientos sociales se produce con la búsqueda del progreso social mediante la eliminación de barreras que dificultan la inclusión, el apoyo y la ayuda a colectivos debilitados o que carecen de voz propia, y la mitigación de los efectos secundarios indeseables de la actividad económica, en definitiva, ayudando al beneficiario a obtener un valor que, por diversos motivos, habrían estado fuera de su alcance [...] Si bien todas las empresas sociales nacen con el fin de generar valor social, no todas comparten la meta de obtener retornos económicos, lo que da lugar a la existencia de empresas no lucrativas y empresas híbridas (Marchante y Sánchez, 2015, p. 182).

Un valor sociali que debe ser vinculado a la justicia social, no a conceptualizaciones esencialistas e inmovilista, al contrario, la reconstrucción ética y solidaria del sentido social de la educación debe potenciarse, situando la práctica educativa, al alumnado y al docente como agentes para el cambio social; precisamente, en la actualidad se están produciendo transformaciones sociales y desplazamientos en los agentes educativos, en el rol del profesorado, en el papel de las instituciones educativas y de los modos de socialización. Consecuentemente, resignificar los escenarios educativos a través de la construcción de justicia social no comprenderá borrar las diferencias de cada colectivo, sino reconocer que dicho proceso encontrará su camino en la medida que exista una transformación en la manera de concebir la igualdad entre grupos social y culturalmente diferentes (Young, 2002). Será necesario, pues, desde la perspectiva educativosocial indagar en una justicia social reflexiva, pluralii, crítica y compleja (Fraser, 2008). 
Un valor social sostenible que se afirma en el bien público y común, en la eliminación de barreras, los procesos estigmatizantes y de exclusión, "el apoyo y la ayuda a colectivos debilitados o que carecen de voz propia, y la mitigación de los efectos secundarios indeseables de la actividad económica" (Marchante y Sánchez, 2015, p.182).

El eje común en las distintas conceptualizaciones de emprendimiento social consiste en la búsqueda de soluciones a problemáticas sociales y en la articulación de "emprendimientos" para solventarlos, restringirlos y transformarlos (Vázquez y Dávila, 2008). Según Martin y Osberg (2007, p. 35) será posible establecer tres componentes en las definiciones del emprendimiento social: (1) la identificación de sectores y sistemas sociales injustos, causantes de exclusión y sufrimientos a colectivos tradicionalmente vulnerables; (2) identificar una oportunidad en este equilibrio injusto, desarrollando una propuesta de valor social desde nuevos imaginarios políticosociales, desde la acción directa, las fortalezas y el desafío a lo hegemónico; (3) concebir y moldear nuevos equilibrios y la creación de un ecosistema estable alrededor que asegure futuros posibles y "mejores" para colectivos y para la sociedad en su diversidad.

La innovación desde y para la social posiciona y equilibra, entonces, al sujeto como agente consciente de las causas y orígenes de las desigualdades, de los desajustes e injusticias. Asimismo, tal y como expone Martínez-Celorrio (2017): "la innovación social hay que entenderla como una geometría variable y calidoscópica de iniciativas y respuestas que admite muchas configuraciones" (p. 63). Desde la perspectiva de la participación social las últimas dos décadas ha sido cruciales para vislumbrar nuevos modos de intercambio de información, de generación de conocimiento, para proyectar novedosos espacios de socialización que han posibilitado una descentralizado de ciertas relaciones de poder, de reconsideración de toma de decisiones públicas y de participación directa siempre con resultados desiguales (Martínez-Celorrio, 2017).

Por otro lado, será fundamental desde la perspectiva educativosocial ampliar las nociones tradicionalmente anquilosadas sobre inclusión, así pues, es posible trabajar desde una educación inclusiva que deje de pensarse a partir de sujetos sociológicamente excluidos, asumiendo que la injusticia y las múltiples formas de vulneración están presentes en todas las personas y en sus trayectorias sociales y educativas (Kaplan, 2006). En estos nuevos contextos y equilibrios se opera a través de las posibilidades inclusivas del Aprendizaje-Servicio (en adelante ApS) como una herramienta de gran interés educativo para promover la superación de barreras, promover la participación democrática y el desarrollo de competencias (Gallardo, 2017).

Este trabajo articula y describe una experiencia a través de la metodología de ApS en el ámbito de la educación superior a través de la participación de estudiantes de Grado de Educación Social. Si desde la Universidad apostamos por una educación crítica y reflexiva, la introducción de esta metodología que integra el servicio a la comunidad con la enseñanza y la toma de consciencia (Aramburuzabala, 2013) resultará oportuna teniendo en cuenta que hablamos de estrategias que están en continuo movimiento tal y como expondremos en el siguiente epígrafe. Destacar en este sentido, el enfoque a partir de la justicia social -en su dimensión práctica y reflexiva- y desde la perspectiva de un movimiento social transformador, por ello:

[...] los proyectos de ApS con un enfoque de Justicia Social desarrollan debates críticos sobre temas relacionados con el poder, los privilegios y las desigualdades sociales y examinan de forma crítica cuestiones como el racismo y la igualdad de oportunidades, favoreciendo el desarrollo y compromiso social de los participantes desde una perspectiva transformadora. Los estudiantes reflexionan de forma estructurada sobre estas realidades, su origen, cómo prevenirlas y afrontarlas, y sobre el impacto del servicio en la mejora de la situación de injusticia y en el cambio social (Aramburuzabala, 2013, p. 7). 
Una metodología para la acción: Aprendizaje-servicio (ApS) y sus implicaciones didácticas. Del aula a la comunidad

En un primer punto, será pertinente definir la metodología ApS como una metodología para la acción que circula del aula a la comunidad y viceversa, también como una metodología en permanente movimiento lo cual beneficia a la propia reflexión sobre la misma en cuanto a su impacto en la comunidad. Autoras como Aramburuzabala (2013) presentan el ApS como una herramienta de gran valor para el aprendizaje y la transformación social que "permite que los estudiantes aprendan mientras actúan sobre las diversas necesidades del entorno con el objetivo de transformar la realidad mediante acciones de mejora, al tiempo que reflexionan de forma estructurada sobre la experiencia vivida" (Aramburuzabala, 2013, p. 6). Es en esa participación en la planificación e implementación de proyectos sociales en la que el alumnado aprende y demuestra competencias en la acción (Tejedor et al., 2019).

Se llevan articulando y desarrollando proyectos de ApS en diversos contextos a lo largo de las dos últimas décadas (Arratia, 2008; Rodríguez-Gallego, 2014). Contextos condicionados por las nuevas dinámicas materiales, políticas y sociales que concretando en educación superior implica retos como el incremento del número de alumnado matriculado o la diversidad del mismo (Mahasneh, Tawalbeh, Al-Smadi, Ghaith y Dajani, 2012). Asimismo, cabe añadir a este punto que en los últimos años la Universidad como institución ha sido "cercada" (Hernández et al., 2013) a través de políticas tecnócratas en busca del exclusivo beneficio económico, la precarización del profesorado e investigador y el alejamiento y desconocimiento del alumnado de su realidad social próxima. Este hecho se complementa con estudios recientes en los cuales se evidencia la falta de compromiso social de los titulados, e incluso cómo este compromiso social disminuye a medida que el alumnado avanza en la carrera universitaria (Segalàs, Ferrer y Mulder, 2010; Cech y Sheric, 2015; citado en Segalàs y Sánchez, 2019).

El ApS se traduce en la apropiación de una serie de saberes y conocimientos por parte de la comunidad. Un camino que transita a través de formas de aprendizaje activo e integrado en el currículo, en el que las alumnas y alumnos participan activamente en experiencias de servicio organizadas para adquirir conocimientos y cubrir necesidades sociales. Un aprendizaje desde y para la experiencia en el cual el compromiso es fundamental junto al desarrollo de competencias profesionales (Rodríguez-Gallego, 2015, p. 315). Una responsabilidad social basada a su vez en un compromiso con valores inclusivos, cimentados en una perspectiva de derechos imprescindible para preparar al alumnado en la participación activa en modos de vida sostenibles, en comunidades y entornos sostenibles tanto local como globalmente (Echeita y Navarro, 2015). Una herramienta para educar sobre y hacia la sostenibilidad; que es en sí misma una experiencia sostenible orientada a la promoción del desarrollo humano sostenible (Aramburuzabala, Cerrillo y Tello, 2015, p.79).

Esta orientación práctica y comprometida en iniciativas de transformación social es la base que justifica su potencial para favorecer procesos de inclusión, capacitar y empoderar al alumnado, a la vez que se refuerza su autoestima al asumir un rol activo y protagonista en la acción social (Mendía y Moreno, 2010). Hablamos, por tanto, de "humanizar" la educación y lo social (Fernández-Maillo, 2019) en un sistema educativo acusado de mantenerse "ajeno a las necesidades sociales" (Aramburuzabala, 2014; citado en Aramburuzabala, Cerrillo y Tello, 2015, p.85), para promover aprendizajes prácticos, adaptativos y coherentes con las demandas actuales de la sociedad en entornos colaborativos de participación y corresponsabilidad. 
El ApS es innovador en la forma en la que el estudiante construye conocimiento mediante necesidades reales de su entorno próximo, relacionando sus procesos y actividades de aprendizaje con la construcción de una ciudadanía activa (Martínez-Martín, 2008). Encontramos, pues, una metodología innovadora en su doble dimensión: la pedagógica y la social (Mayor, 2019b); comprende, entonces, una sincronía entre "la intencionalidad pedagógica y la intencionalidad social, mejorando así la calidad de los aprendizajes en tanto se articula teoría y práctica y planifica una respuesta participativa a una necesidad social" (Montes, Tapia y Yaber, 2011, p. 42). Esto requiere promover la experiencia real de los protagonistas del proceso de enseñanza-aprendizaje en una actividad asociada a una proyección social cuyo beneficiario es el propio entorno social (Dewey, 1924, citado en Mayor, 2019b), siendo esta a su vez requisito para promover la plena integración en la sociedad de cada nueva generación.

De acuerdo a Tejedor el al. (2019), son cinco las condiciones características de una experiencia de ApS:

1) abordar las necesidades particulares de la comunidad, que son integradas en los objetivos curriculares que favorecen la comprensión crítica y el desarrollo del razonamiento moral en los estudiantes;

2) relaciones interpersonales entre el alumnado, en donde las entidades de la comunidad y las personas profesionales con quienes interactúan se caracterizan por el reconocimiento, el respeto mutuo y la simetría;

3) las tareas tienen un carácter colaborativo y permiten tiempo para la reflexión individual;

4) se requiere un análisis de los valores y contravalores del contexto institucional en el que se desarrollan; $y$

5) todos los participantes participan en la evaluación.

\section{Contextualizaciones de la experiencia: el Tercer Sector de Acción Social (TSAS)}

El modelo de Estado de Bienestar en España se ha caracteriza por una complementariedad entre la acción social del Estado y la sociedad civil a través de las entidades de acción social (Fernández- Maíllo, 2019). Estas entidades configuran el llamado Tercer Sector (en adelante TS), compuesto a su vez de dos subsectores: la economía socialiii (cooperativas, mutuas, sociedades laborales, cofradías de pescadores y nuevas formas de economía colaborativa y solidaria surgidas en el proceso de la crisis financiera) y, por otra parte, las ONG (compuesto principalmente por asociaciones y fundaciones, y dentro del cual destaca el Tercer Sector de Acción Social (en adelante TSAS)iv) (Fernández- Maíllo, 2019). Durante la década de la crisis y la postcrisis (2008-2018), el régimen de bienestar español se ha visto alterado en todas sus esferas (Fernández- Maíllo, 2019), afectando significativamente a todos los agentes sociales. Entre ellos, el TS y, especialmente el TSAS, ha tenido que potenciar aún más su función prestacional como proveedor de servicios de protección social para dar respuesta a las necesidades sociales, manteniendo sus tradicionales funciones reivindicativas de abogacía, representación y sensibilización social (Marbán, 2014). Como ya venían denunciando los documentos de trabajo del VII Informe sobre exclusión y desarrollo social en España, el reto de tener que hacer frente a una mayor demanda de atención, en un entorno más vulnerable y con menos recursos para darles respuestav ${ }^{v}$, ha supuesto una crisis de sostenibilidad y la obligada recalibración del sector a nivel organizativo interno y externo (Marbán, 2014).

A nivel global, estos cambios y la necesidad de asunción de responsabilidades compartidas se encuadran en un contexto internacional de preocupación por responder a los desafíos mundiales actuales y lograr el desarrollo humano sostenible. Dentro de la Agenda 2030 
sobre el Desarrollo Sostenible, aprobada por la ONU en 2015, la creación de alianzas entre los agentes sociales es fundamental. En concreto, la meta 17.17 indica la necesidad de fomentar y promover la constitución de alianzas eficaces en las esferas pública, público-privada y de la sociedad civil, aprovechando la experiencia y las estrategias de obtención de recursos de las alianzas (Red Española del Pacto Mundial de Naciones Unidas, 2019). Esto implica que, desde el TS Social, se deba asumir plenamente su papel esencial en el camino hacia el Desarrollo Humano Integral y Sostenible. Así como, desde la Educación Superior (SDSN Australia/Pacific, 2017) debemos: 1) Dotar al alumnado de conocimientos, habilidades y motivación para entender y abordar los Objetivos de Desarrollo Sostenible (en adelante ODS), 2) Empoderar y movilizar a la juventud para ser los agentes activos del desarrollo sostenible, 3) Proporcionar amplia formación académica o vocacional para implementar soluciones de ODS y 4) Crear más oportunidades para la creación de capacidades de estudiantes y profesionales de países en desarrollo para abordar los desafíos relacionados con los ODS.

En definitiva, radica en una llamada a la responsabilidad colectiva y al trabajo sinérgico de todos los implicados en una alianza hacia la transformación que responde al imperativo de “...recrear, redescubrir y regenerar los vínculos, los valores y el tejido institucional. Para eso necesitamos un nuevo gran movimiento humanista de la mayoría de la sociedad para fortalecer los sujetos sociales, el relato común compartido y la institucionalidad que haga posible el camino de un Desarrollo Humano Integral" (Fernández-Maíllo, 2019, p. 94).

\section{Sostenibilización curricular de la educación superior para la profesionalización y empleabilidad de agentes de transformación y cambio social. Tercer Sector como ámbito profesional de la educación social}

Desde la Educación Superior y, en concreto, desde el campo de la Educación Social, debemos hacer una doble lectura de esta situación en lo que respecta a las demandas de la sociedad actual. Por un lado, la necesidad de preparar a los y las profesionales que la sociedad necesita en cada momento y sector y, por otro, favorecer la inserción laboral y la empleabilidad del alumnado universitario en un contexto de inseguridad laboral -incluyendo niveles de empleo/desempleo, así como de subempleo- (García-Blanco y Cárdenas-Sempértegui, 2018).

A esto se suma la necesidad de introducir la Educación para el Desarrollo Sostenible (en adelante EDS) para mejorar la formación de los y las futuros profesionales, como vía de capacitación para afrontar los retos que plantea la actual situación global. Es decir, iniciar un proceso de Sostenibilización curricular (CRUE, 2005).

A nivel institucional-organizativo, sostenibilidad el currículum entraña la "reconceptualización del conjunto de la institución universitaria a la luz de los valores, objetivos, formas de gestión e iniciativas que implican un mayor compromiso con la sociedad y con la contribución a un nuevo modelo de desarrollo más equilibrado y sostenible" (Comisión Técnica de la Estrategia Universidad 2015, 2011, p. 11). A nivel formativo-competencial, "implica el empoderamiento de la comunidad universitaria y la creación de espacios para la reflexión y la colaboración colectiva, inter y transdisciplinaria, que fomenten el aprendizaje, la reflexión crítica sobre las prácticas y cosmovisiones existentes y la acción creativa e innovadora" (Segalàs-Coral y Sánchez-Carracedo, 2019, p. 1204-3). En definitiva, se trata de que la Universidad eduque profesionales como agentes de transformación y cambio social, a la vez que forme a ciudadanos con conciencia ética y compromiso cívico vinculando la sostenibilidad a esta formación (Michelsen, 2016; citado en Serrate-González et al, 2019; Tejedor et al., 2019). 
Tomando las palabras de Mindt y Rieckmann (2017), el desarrollo sostenible demanda nuevas formas de organizar las economías (incluida la economía social), compuestas de organizaciones resilientes que contribuyan al mantenimiento de ecosistemas saludables y promuevan la justicia social. Por tanto, se requieren personas emprendedoras con orientación a la sostenibilidad, es decir, personas con el potencial de crear soluciones para los múltiples desafíos que enfrentan las crisis ecológicas, sociales, políticas y financieras (Lintner et al., en revisión; citado en Mindt y Rieckmann, 2017). Para ello, la educación universitaria, así como en el resto de niveles educativos, debe promover el desarrollo de competencias transversales clave para la sostenibilidad. Hasta la fecha, varias investigaciones han identificado competencias clave relevantes para el desarrollo sostenible, sin embargo, no existe una única clasificación de competencias para la sostenibilidad. A nivel internacional, la UNESCO (2017) señala competencias tales como las competencias de pensamiento sistémico, de anticipación (o pensamiento futuro), normativa (o pensamiento de valores), estratégica (u orientada a la acción creativa e innovadora), de colaboración (sociales o interpersonal), de pensamiento crítico (críticoreflexivas), de autoconciencia y la competencia integrada a la resolución de problemas (metacompetencia para aplicar las anteriores en la solución de problemas de sostenibilidad y fomentar el desarrollo sostenible) (UNESCO, 2017; Wiek et al., 2011, 2016; citado en Mindt y Rieckmann, 2017). A nivel nacional, la Comisión Sectorial de la CRUE en Sostenibilidad (CADEP-CRUE, 2012) definió cuatro competencias que, a pesar de requerir actualización, pueden servirnos de marco general desde el cual desarrollar las propuestas concretas aplicadas a cada titulación, como están haciendo desde el proyecto EDINSOST (Segalàs y SánchezCarracedo, 2019; Serrate et al, 2019; Tejedor et al, 2019). Estas son:

- C1: Contextualización crítica del conocimiento estableciendo interrelaciones con la problemática social, económica y ambiental, local y/o global.

- C2: Utilización sostenible de recursos y prevención de impactos negativos sobre el medio natural y social.

- C3: Participación en procesos comunitarios que promuevan la sostenibilidad.

- C4: Aplicación de principios éticos relacionados con los valores de la sostenibilidad en los comportamientos personales y profesionales.

Dichas competencias transversales, multifuncionales e independientes del contexto, deben sumarse a las competencias específicas de cada situación y contexto (UNESCO, 2017). Cada Universidad, por tanto, debe integrar estas competencias y los principios de la Educación para el Desarrollo Sostenible (EDS) en la formación académica y vocacional (SDSN Australia/Pacific, 2017). Desde el punto de vista metodológico, la sostenibilización curricular requiere "una pedagogía transformadora y orientada a la acción, y se caracteriza por aspectos tales como el aprendizaje autodidacta, la participación y la colaboración, la orientación hacia los problemas, la inter y transdisciplinariedad, y la creación de vínculos entre el aprendizaje formal e informal" (UNESCO, 2017:7).

Hablamos, pues, de un enfoque de enseñanza-aprendizaje basado en competencias y centrado en el alumnado, que promueva un aprendizaje activo, reflexivo, colaborativo, experiencial, interdisciplinar y en conexión o aplicado en escenarios reales (Mindt y Rieckmann, 2017; Litzner y Rieß, 2019; Tejedor et al., 2019) desde la triple perspectiva del desarrollo sostenible: ambiental, social y económica (Aznar y Barrón, 2017; Segalàs-Coral y SánchezCarracedo, 2019). Entre las metodologías más afines, podemos destacar las analizadas por el proyecto EDINSOST, entre ellas cabe destacar: Aprendizaje basado en problemas; Aprendizaje orientado a proyectos; Aprendizaje-Servicio; Simulación y Estudio de Casos (Segalàs y Sánchez, 2019; Tejedor et al. 2019). 
Por este motivo, se consideran métodos clave (UNESCO, 2017, p. 55):

- Proyectos colaborativos del mundo real, tales como proyectos de aprendizaje y servicio y campañas para distintos ODS.

- Los ejercicios de construcción de visión, tales como talleres de futuro, análisis de escenario, narrativa utópica/distópica, pensamiento de ciencia ficción, y pronóstico y retrospección.

- Análisis de sistemas complejos por medio de proyectos de investigación basados en la comunidad, estudios de caso, análisis de partes interesadas, análisis de actores, modelado, juegos de sistemas, etc.

- El pensamiento crítico y reflexivo mediante debates en "pecera", diarios de reflexión, etc.

\section{La figura del educador social: redefiniciones y competencias}

Unida a las cambiantes demandas y necesidades sociales, la profesión de educador y educadora social debe renovarse permanentemente para darles respuesta (Ruiz, Martín y Cano, 2015). En coherencia con el entorno social complejo, dinámico y cambiante en el que desarrolla su labor profesional (Herrera, 2010; citado en Ruiz, Martín y Cano, 2015), la definición de la educación social y sus competencias profesionales deben ser dinámicas, flexibles y polivalentes (Eslava, 2019).

En este sentido, encontramos propuestas como la desarrollada por Eslava (2019) en su Tesis "Diseño del perfil competencial de la profesión de la educación social", a partir de la cual desarrolla una propuesta renovada de perfil competencial del educador y la educadora social, y que ajusta la formación inicial de la profesión a la práctica real. Este modelo de formación basado en competencias se presenta como garante de una formación inicial generalista universitaria, fundamentado en el desarrollo de competencias específicas definitorias de la profesión, favorecedoras de la empleabilidad, cuya especialización y satisfacción de necesidades formativas se canaliza desde la formación permanente.

Contribuciones como la llevada a cabo por Eslava (2019), pueden dar una respuesta ágil y adaptativa a la incertidumbre vivida en los últimos años en la Educación Social, debido a las políticas sociales intervencionistas, los recortes presupuestarios (como factor debilitante del Estado de Bienestar según Fernández- Maíllo, 2019) y el aumento de la inseguridad en muchas entidades y profesionales. A este respecto, las entidades del TS, espacio tradicional de trabajo de la Educación Social, ha vivido un momento de crisis, seguida de una obligada recalibración que ha supuesto la consolidación de su legitimidad como prestador de protección social y se han profesionalizado, estructurándose y aplicando modelos de gestión que les han permitido ser más eficientes y competitivos en el sector (Álvarez, 2017; Marbán, 2014).

Teniendo en cuenta que la profesionalización se materializa en la práctica profesional, la formación inicial de los Educadores y Educadoras Sociales debe actualizarse para garantizar la adquisición de competencias necesarias para el desempeño profesional que sean útiles, aplicables y ajustadas al tipo de ámbito en un marco social cada vez más imprevisible y dinámico (Lizarte y Fernández, 2018), en el caso que nos ocupa el TS. Esta será la vía, como indicamos anteriormente, para favorecer la inserción laboral y la empleabilidad de nuestro alumnado universitario en un contexto de cambios e inseguridad.

En este sentido, estudios recientes están demostrando la importancia de orientar la formación superior basada en competencias hacia:

- Los factores actuales y contextualizados de ocupabilidad o condiciones de búsqueda y acceso al empleo vinculados al contexto, es decir, el proceso de inserción laboral (Fernández-Salinero y García-Álvarez, 2019; García-Blanco y Cárdenas-Sempértegui, 2018). 
- La competencia de la persona para acceder al empleo e integrarse en el mercado laboral respondiendo adecuadamente a lo que se requiere, es decir, la empleabilidad (GarcíaBlanco y Cárdenas-Sempértegui, 2018; García-Gutiérrez, 2014; citado en FernándezSalinero y García-Álvarez, 2019). Especialmente importantes en este sentido son las competencias interpersonales como: "la capacidad crítica y autocrítica, el trabajo en equipo, las habilidades socio-relacionales, la capacidad para trabajar interdisciplinarmente o la de comunicarse con expertos de otras áreas resultan decisivas para incrementar el capital interpersonal del alumnado y capacitarlo para la búsqueda de empleo" (Fernández-Salinero y García-Álvarez, 2019, p. 182)

- Junto a estas competencias para la empleabilidad podríamos considerar las competencias para el emprendimiento. Estas albergarían aquellas habilidades y destrezas personales para poner en marcha iniciativas, diseñar nuevos proyectos y/o generar ideas innovadoras, es decir, el emprendimiento (Fernández-Salinero y De la Riva, 2014; citado en FernándezSalinero y García-Álvarez, 2019:166).

Frente a planteamientos tradicionales profesionalizadores basados en la especialización, tanto desde la perspectiva de la empleabilidad como del desempeño profesional, se está poniendo de relieve la importancia de las competencias transversales interpersonales, incluso por encima de las competencias específicas relacionadas con los conocimientos disciplinarios propios de la titulación, tanto por el alumnado como por profesionales de la Educación Social (Lizarte y Fernández, 2018). Parece claro poder afirmar entonces, la existencia de la necesidad de reincidir en la promoción de la formación en competencias transversales, multifuncionales e independientes del contexto (UNESCO, 2017), que sumadas a las competencias específicas disciplinares y funcionales de acuerdo con Eslava (2019)- contribuyan a la inclusión y a la inserción laboral de profesionales emprendedores/as, comprometidos/as socialmente y con competencias profesionalizadoras orientadas a la sostenibilidad.

\section{Diseño de la experiencia para el Grado en Educación Social (UEX)}

La experiencia de ApS se articula en el marco de la asignatura de Organización y Gestión de Servicios Educativo-Sociales, cursada por el alumnado de tercer curso del Grado de Educación Social de la Facultad de Formación del Profesorado de Cáceres (Universidad de Extremadura) durante el primer semestre de los cursos académicos 2018-2019 y 2019-2020. Esta ha sido motivada por los buenos resultados en cuanto a la satisfacción del alumnado en experiencias anteriores desarrolladas en la misma asignatura, y la demanda expresa del alumnado por acercarse a la realidad de las instituciones del ámbito profesional de la Educación Social (Mayor, 2019a).

La participación en dicha experiencia ha sido efectuada por las 69 alumnas y alumnos matriculados en la asignatura durante el curso 2018-2019 y 66 en 2019-2020, los cuales, a través del trabajo en diversos equipos han puesto en funcionamiento la labor de investigadores y asesores de entidades sociales del entorno. Concretamente, el reto o propósito del servicio a través del cual se estructura la experiencia consistía en: reforzar la capacidad de gestión y de respuesta resiliente de las entidades sociales del entorno, al mismo tiempo que se ampliaba su capacidad para hacer frente a los cambios sociales y económicos que, a su vez, influyen en su capacidad para dar respuesta a los problemas sociales que forman parte de su misión y visión. Para ello, el alumnado debe aprender a gestionar y coordinar entidades, equipamientos y grupos, de acuerdo a los diferentes contextos y necesidades (Competencia CE13 del Grado).

El servicio orientado a cubrir la necesidad social o reto de la experiencia ha consistido en el desarrollo de un Plan Estratégico de Acción, personalizado y coherente con la identidad propia de una organización del contexto local, orientado a mejorar la calidad en la gestión de la 
organización y a potenciar su capacidad de adaptación permanente al medio, con el fin último de reforzar el TSAS como prestador de servicios sociales. De acuerdo a la clasificación de modalidades de actuación mediante el ApS de Zorrilla, Capella y Gil (2016), podemos definir el servicio como indirecto, ya que el alumnado debe trabajar desde y para las entidades sociales sin entrar en contacto directo con la población a la que atienden; y de investigación, ya que se basa en el análisis situacional de la entidad para que les permita llevar a cabo un Plan Estratégico de mejora de la organización y, con ello, valorar su capacidad de respuesta a las necesidades sociales de sus destinatarios y destinatarias y del entorno.

El proceso de definió en la siguiente secuencia: 1) acercamiento a la entidad y documentación a través de una entrevista con trabajadores o trabajadoras de la entidad elegida y revisión documental ,2) diagnóstico estratégico organizacional a través del análisis interno y externo y la priorización de estrategias resilientes organizativas y de gestión (análisis DAFOCAME), y 3) aplicación de herramientas para la planificación estratégica (p.e., Metodología del Marco Lógico, Cuadro de Mando Integral, ...) para guiar el proceso de valoración de alternativas y toma de decisiones en cuanto a la solución estratégica y 4) redacción de informe final para la entidad con la priorización de soluciones de mejora y la valoración de su impacto, tanta para la propia organización como para el colectivo destinatario al que atiende.

Siguiendo a Rubio (2008), los aprendizajes vinculados al servicio pueden resumirse en:

- Aprender a conocer: la complejidad y riqueza del contexto comunitario (asociaciones y personas comprometidas en la transformación social). El alumnado se acerca a una entidad social del entorno, desde la cual conocerá su misión, visión y valores, así como los aspectos relacionados con su gestión y situación contextualizada.

- Aprender a hacer: competencias específicas del servicio que se realiza (diseño, comunicación, difusión). El alumnado, através de las fases de la secuencia de aprendizaje, aprende a aplicar distintas herramientas de planificación y pensamiento estratégico para la gestión.

- Aprender a ser: compromiso y responsabilidad, esfuerzo y constancia, tolerancia a la frustración. A través del conocimiento y reconocimiento de la labor de las entidades sociales del TS en el sistema de bienestar social, así como la asunción de un rol activo y protagonista en la resolución de problemas reales de la entidad con impacto para la misma y las personas a las que atiende.

- Aprender a convivir: sentimiento de pertenencia a la comunidad, hábitos de convivencia (comprensión, amabilidad, paciencia, generosidad, solidaridad), compromiso, responsabilidad y participación en la comunidad y cuestiones públicas. El alumnado desarrolla competencias interpersonales para el trabajo colaborativo a través de la propia dinámica de trabajo grupal en el aula, y en la interacción con la entidad social.

- Aprender a emprender: planificar, tomar decisiones, actuar, evaluar lo hecho y autoevaluarse, buscar soluciones y llevarlas a la práctica. Hablamos, por tanto de, competencias relacionadas con el emprendimiento, imprescindibles para crear e implementar soluciones orientadas al desarrollo sostenible (Lintner et al., en revisión; citado en Mindt y Rieckmann, 2017; SDSN Australia/Pacific, 2017), movilizadas a través de la "acción creativa e innovadora" (UNESCO, 2017; Segalàs-Coral y Sánchez-Carracedo, 2019: 1204-3). El alumnado, durante el proceso, tiene que crear soluciones creativas e innovadoras para resolver los problemas que enfrenta la entidad en la que centran su atención y, con ello, promover la sostenibilidad de las mismas asegurando su contribución a la protección y bienestar social. 


\section{CONCLUSIONES PROVISIONALES Y LIMITACIONES DE LA EXPERIENCIA}

La nueva realidad que se nos presenta demanda que la Educación Superior se adapte, humanice y dinamice adoptando como propia "una pedagogía transformadora y orientada a la acción" (UNESCO, 2017:7). En este proceso de sostenibilización curricular, acercarse a la realidad y el resto de agentes sociales que en ella operan, para dotar con competencias "útiles, aplicables y ajustadas" (Lizarte y Fernández, 2018) a los ciudadanos y ciudadanas del presente y del futuro para que participen de forma comprometida en el desarrollo sostenible de la sociedad.

Desde las distintas ópticas, niveles y ámbitos, parece existir un consenso en cuanto a la necesidad de mejorar la formación de las y los futuros profesionales, orientando la formación basada en competencias hacia el desarrollo humano integral y sostenible, como vía de capacitación para afrontar los retos que plantea la actual situación global (CRUE, 2015). Lo que a su vez, implica "la formación de ciudadanos más competentes para la vida del siglo XXI a través de la contextualización de los aprendizajes" (Mendía, 2017; citado en Solís y López, 2019), para lo cual la metodología de ApS está demostrando ser una de las estrategias didácticas más adecuadas y eficaces.

La participación por parte del alumnado y la toma de consciencia se sitúan como ejes esenciales en este tipo de proyectos. Por ellos, el conocimiento profundo de las problemáticas sociales próximas activa la transferencia entre espacio educativo y espacio social; añadido a una especial sensibilización por "otras realidades", por la diversidad, el diálogo con el "otro" y de la autorregulación en la construcción y generación de los aprendizajes experienciales. Es importante también destacar sobre este tipo de experiencias como el estudiante percibe una retroalimentación próxima en el tiempo, una resonancia de las problemáticas que repercuten en su entorno inmediato, por lo que se establece una implicación del estudiante como ciudadano activo con su comunidad con la que consecuentemente establece alianzas y redes de apoyo, profesionales y personales.

Esta experiencia de enseñanza-aprendizaje se plantea con un diseño emergente, por lo que se requieren distintos ciclos de diseño y rediseño orientados a la toma de decisiones en cuanto a la mejora de su eficacia y eficiencia. Dado el potencial descriptivo de este trabajo resultará de interés para futuras publicaciones explorar los resultados en cuanto a la evaluación de resultados e impacto de la experiencia en las siguientes dimensiones: "desarrollo académico y cognitivo; desarrollo cívico; desarrollo vocacional y profesional; desarrollo ético y moral; desarrollo personal y desarrollo social" (Ruiz-Corbella y García-Gutiérrez, 2019; Tapia, González y Elicegui, 2005; Uruñuela, 2018; citado en Mayor, Lòpez y Solís, 2019, p. 159). En lo que respecta al perfil competencial y los aprendizajes, será interesante investigar sobre las competencias asociadas a la innovación social y a la resolución de problemas (BEPA 2010; citado en Fernández- Maíllo, 2019), como las competencias relacionadas con el emprendimiento social por su incidencia en la capacidad del alumnado para transformar la realidad social.

\section{BIBLIOGRAFÍA}

Álvarez, A. (2017). El desempeño profesional del educador y la educadora social: funciones, competencias y creencias de autoeficacia. (Tesis doctoral). Madrid, España: Universidad Autónoma de Madrid.

Aramburuzabala, P. (2013). Aprendizaje-servicio: Una herramienta para educar desde y para la justicia social. Revista Internacional de Educación para la Justicia Social 2 (2) 5-11.

Aramburuzabala, P., Cerrillo, R., \& Tello, I. (2015). Aprendizaje-servicio: una propuesta metodológica para la introducción de la sostenibilidad curricular en la universidad. Profesorado. Revista de Currículum 


y Formación de Profesorado, 19(1), 78-95. Recuperado de:
https://www.ugr.es/ recfpro/rev191ART5.pdf

Arratia, A. (2008): «Ética, solidaridad y aprendizaje servicio en la educación superior». Acta Bioethica, Vol. $14, N^{\circ} 1$.

Aznar, P. y Barrón, Á. (2017). El desarrollo humano sostenible: un compromiso educativo. Teoría De La Educación. Revista Interuniversitaria, 29(1 (en-jun)), 25-53. doi:10.14201/teri.16698

Bauman, Z. (2007). Los retos de la educación en la modernidad líquida. Barcelona: Editorial Gedisa.

Beck, U. (1998). La sociedad del riesgo. Barcelona: Paidós.

CADEP-CRUE. (2012). Directrices para la introducción de la Sostenibilidad en el Curriculum. Actualización de la declaración institucional aprobada en 2005. https://www.crue.org/Documentos\%20compartidos/Declaraciones/Directrices Sosteniblidad Crue2 012.pdf

Comisión Técnica de la Estrategia Universidad 2015 (2011) La responsabilidad social de la universidad y el desarrollo sostenible. Madrid: Ministerio de Educación.

CRUE (2005). Directrices para la Sostenibilización Curricular. Documento del grupo de trabajo de Calidad Ambiental y Desarrollo Sostenible.https://www.um.es/documents/10908394/12705734/CRUE sostenibilizacion curricular. pdf/7db00090-d141-430f-988d-6bcb5f4a6e84

Cuenca, R. (2012). Sobre justicia social y su relación con la educación en tiempos de desigualdad. Revista Internacional de Educación para la Justicia Social, 1(1). 79-93

Echeita, G. y Navarro, D. (2015). Educación inclusiva y desarrollo sostenible. Una llamada urgente a pensarlas juntas. Edetania, 46, 141-162.

Eslava, M. D. (2019). Diseño del perfil competencial de la profesión de la educación social. (Tesis doctoral). Córdoba, España: Universidad de Córdoba.

Fernández- Maíllo, G. (Coord.) (2019). VIII Informe sobre exclusión y desarrollo social en España. Madrid: Fundación Foessa. Recuperado de: https://www.foessa.es/viii-informe/

Fernández-Salinero, C. y García-Álvarez, J. (2019). La inserción laboral de graduados y graduadas a través de los contactos personales. Una propuesta desde la gestión del conocimiento. TeoríA De La EducacióN. Revista Interuniversitaria, 32(1(en-jun)), 163-189. doi:10.14201/teri.20196

Fraser, N. (2008). Escalas de justicia. Barcelona: Herder.

Fundación Zerbikas.

Gaete, R. (2015). El voluntariado universitario como ámbito de aprendizaje servicio y emprendimiento social: Un estudio de caso. Última década, 23(43), 235-260.

Gallardo, R. M. (2017). El Aprendizaje-Servicio como una estrategia inclusiva para superar las barreras al aprendizaje ya la participación. Revista de Educación Inclusiva, 5 (1).

García-Blanco, M. y Sempértegui, E. B. C. (2018). La inserción laboral en la Educación Superior. La perspectiva latinoamericana. Educación $X X 1,21(2)$. Recuperado de http://revistas.uned.es/index.php/educacionXX1/article/view/16209

García-Hernández, Y., Mendoza, J, Gutiérrez, G. y Santos, L.E. (2017). Emprendimiento social en estudiantes del área empresarial de una institución de educación superior tecnológica en el Estado de Hidalgo. XVII Congreso Latino-Iberoamericano de Gestión Tecnológica (Altec 2017), Ciudad de México, 16-18 de octubre de 2017.

Hernández, J. (2013). La universidad cercada: testimonios de un naufragio. Barcelona: Anagrama.

Kaplan, C. (2006). La inclusión como posibilidad. Buenos Aires: OEl.

Litzner, L. y Rieß, W. (2019). La Educación para el Desarrollo Sostenible en la universidad boliviana. Percepciones del profesorado. Teoría De La Educación. Revista Interuniversitaria, 31(1 (en-jun)), 149-173. doi:10.14201/teri.19037

López-Rodríguez, J. (2017). La promoción del emprendimiento social mediante metodologías innovadoras: hacia un nuevo paradigma educativo. Lan harremanak: Revista de relaciones laborales, (37), 67-82

Lorey, I. (2016). Estado de inseguridad: Gobernar la precariedad. Madrid: Traficantes de sueños.

Mahasneh, R., Tawalbeh, A., Al-Smadi, R., Ghaith, S., \& Dajani, R. (2012). Integrating service learning in Jordanian higher education. Innovations in education and teaching international, 49(4), 415-425.

Marbán, V. (2014). Tercer Sector de Acción Social y lucha contra la exclusión. VII Informe sobre exclusión y desarrollo social en España. Madrid: Fundación FOESSA. Recuperado de http://www.foessa2014.es/informe/uploaded/documentos trabajo/15102014111516 9007.pdf

Marchante, C. G., \& Sánchez, M. S. M. (2015). El emprendimiento social y el empleo de calidad. Lan harremanak: Revista de relaciones laborales, (32), 180-206.

Martin, R. L., y Osberg, S. (2007). Social Entrepreneurship: The Case for Definition. Stanford Social Innovation Review.

Martínez-Celorrio, X. (2017). La innovación social: orígenes, tendencias y ambivalencias. Sistema. Revista de Ciencias Sociales 247, 61-88.

Martínez-Martín, M. (2008). Aprendizaje servicio y construcción de ciudadanía activa en la universidad: la dimensión social y cívica de los aprendizajes académicos. En Miquel Martínez (Ed.): Aprendizaje servicio y responsabilidad social de las universidades (pp. 11-26). Barcelona: Octaedro. 
Mayor, D. (2019a). El desarrollo de competencias del estudiantado universitario mediante prácticas de Aprendizaje-Servicio: percepción del alumnado. En REDINE (Ed.), Estrategias y metodologías didácticas: perspectivas actuales. (pp. 1-10). Eindhoven, NL: Adaya Press

Mayor, D. (2019b). Dimensiones pedagógicas que configuran las prácticas de aprendizaje-servicio. Páginas de educación, 12(2), 23-42.

Mayor, D., Lòpez, A.M. y Solís, M.G. (2019). El Aprendizaje-Servicio como Escenario Formativo y su Influencia en Distintos Agentes Socioeducativos. Percepción de los Participantes, International Journal of Sociology of Education, 8(2), 153-172. doi: 10.17583/rise.2019.4071

Mendía, R., y Moreno, V. (2010). Guía 3. Aprendizaje y servicio solidario: una estrategia para la inclusión social. Bilbao: Zerbikas Fundazioa.

Mindt, L., y Rieckmann, M. (2017). Desarrollo de las competencias para el emprendimiento orientado a la sostenibilidad en la educación superior: una revisión bibliográfica de los métodos de enseñanza y aprendizaje. Teoría de la Educación. Revista Interuniversitaria, 29 (1 (en-jun)), 129-159. doi:10.14201/teri.16702

Montes, R., Tapia, M., \& Yaber, L. (2011). Manual para docentes y estudiantes solidarios. Buenos Aires: CLAYSS. Recuperado de http://www. clayss. org. ar/04_publicaciones/Natura2013. pdf.

Red Española del Pacto Mundial de Naciones Unidas (2019). Las ONG ante los Objetivos de Desarrollo Sostenible: propuesta de acción del Pacto Mundial. Madrid: Red Española del Pacto Mundial de Naciones Unidas. Recuperado de https://www.pactomundial.org/wp-content/uploads/2019/05/LasONG-ante-los-Objetivos-de-Desarrollo-Sostenible.pdf

Rodríguez-Gallego, M. R. (2015). Una experiencia de aprendizaje-servicio en comunidades de aprendizaje. Profesorado: revista de curriculum y formación del profesorado, 19 (1), 314-333.

Rodríguez-Gallego, M.R. (2014): El aprendizaje-servicio como estrategia metodológica en la universidad. Revista Complutense de Educación, 25 (1). 95-113.

Rubio, L. (2008). Guía zerbikas 0: Aprendizaje y servicio solidario, guía de bolsillo. Bilbao:

Ruiz, M., Martín, A. M., y Cano, M. A. (2015). La consolidación del perfil profesional del educador social: Respuesta al derecho para la ciudadanía. Perfiles educativos, 37(148), 12-19.

SDSN Australia/Pacific. (2017). Cómo empezar con los ODS en las universidades una guía para las universidades, los centros de educación superior y el sector académico. [Getting started with the SDGs in universities: A guide for universities, higher education institutions, and the academic sector.] (Red Española para el Desarrollo Sostenible ed.). Australia, New Zealand and Pacific Edition: Sustainable Development Solutions Network - Australia/Pacific, Melbourne. Recuperado de: http://reds-sdsn.es/quia-empezar-los-ods-las-universidades.

Segalàs, J. y Sánchez, F. (2019). El proyecto EDINSOST. Formación en las Universidades españolas de profesionales como agentes de cambio para afrontar los retos de la sociedad. Revista de Educación $\begin{array}{llll}\text { Ambiental } y & \text { Sostenibilidad, } & \text { Recuperado de }\end{array}$ https://revistas.uca.es/index.php/REAyS/article/view/4784

Serrate, S., Martín, J., Caballero, D., y Muñoz, J. (2019). Responsabilidad universitaria en la implementaci $\tilde{A}^{3} \mathrm{n}$ de los objetivos de desarrollo sostenible. European Journal Of Child Development, Education And Psychopathology, 7(2), 183-196. doi:10.30552/ejpad.v7i2.119

Solís, M.G., y López, A.M. (2019). Hacia la consolidación del Aprendizaje-Servicio como metodología innovadora para la mejora educativa-social en Extremadura. En REDINE (Ed.), Estrategias y metodologías didácticas: perspectivas actuales. (pp.30-40). Eindhoven, NL: Adaya Press.

Tejedor, G., Segalàs, J., Barrón, Á., Fernández-Morilla, M., Fuertes, M. T., Ruiz-Morales, J., ... \& Hernández, À. (2019). Didactic strategies to promote competencies in sustainability. Sustainability, 11(2086), 124. doi: https://doi.org/10.3390/su11072086

Vásquez, A. G., \& Dávila, M. A. T. (2008). Emprendimiento social-revisión de literatura. Estudios gerenciales, 24(109), 105-125.

Young, I. M. (2000). La justicia y la política de la diferencia (Vol. 59). Universitat de València.

Zorrilla, L., Capella, C., y Gil, J. (2016). Aprendizaje-servicio. En O. Chiva y M. Martí (Coords.), Métodos pedagógicos activos y globalizadores. Conceptualización y propuestas de aplicación. (1ª ed. pp. 6783). Barcelona: Graó

i “La creación de valor social sostenible es la característica clave que diferencia el emprendimiento social de las obras de beneficencia o las actuaciones caritativas de individuos bien intencionados" matizan Vázquez y Dávila (2008, p. 108).

ii Véase el trabajo Cuenca (2012) en el cual revisa perspectivas diversas acerca de la necesidad de reconocer la pluralidad de la conceptualización de la justicia social: "Esta pluralidad supone reconocer que la justicia social es una categoría que transita entre varias dimensiones, que es menos "objetiva" de lo que se pensaba y que acepta múltiples formas de ser tratada. Por lo tanto, la pluralidad de la justicia social renuncia en cierta forma al halo normativo-jurídico 
que la mantuvo contenida durante muchos años para cederle paso a un marco en el que los asuntos políticos se encuentran con los culturales y los económicos" (Cuenca, 2012, p. 84).

iii Artículo 5.1 de la Ley 5/2011, de 29 de marzo, de Economía Social (LES).

iv Las entidades del TSAS son definidas como "aquellas organizaciones de carácter privado, surgidas de la iniciativa ciudadana o social, bajo diferentes modalidades, que responden a criterios de solidaridad y de participación social, con fines de interés general y ausencia de ánimo de lucro, que impulsan el reconocimiento y el ejercicio de los derechos civiles, así como de los derechos económicos, sociales o culturales de las personas y grupos que sufren condiciones de vulnerabilidad o que se encuentran en riesgo de exclusión social" (Ley 43/2015, de 9 de octubre, del Tercer Sector de Acción Social , Artículo 2.1.).

$\checkmark$ Tal como se indica en el último Informe FOESSA: "haciendo más trabajo con menos recursos, ajustando algo sus plantillas por los recortes del gasto social, aumentando la contribución del voluntariado y aportando más recursos propios" (Fernández- Maíllo, 2019:327). 